\title{
Sistema constructivo con Guadua aculeata para la producción social de la vivienda.
}

\section{Construction system with Guadua aculeata for the housing social production}

\author{
Alvaro Hernández Santiago(1) \\ Diego Torres Hoyos ${ }^{(1)}$ \\ (1) Facultad de Arquitectura, \\ Universidad Veracruzana, Poza Rica, \\ Veracruz, México. \\ alvahernandez@uv.mx \\ dtorres@uv.mx
}

Recibido: 7 de Julio, 2019

Aceptado: 10 de Noviembre, 2019

Doi: http://dx.doi.org/10.24133/ciencia. v22i1.1290

"THIS IS AN OPEN ACCESS ARTICLE UNDER THE TERMS OF THE CREATIVE COMMONS ATTRIBUTION LICENSE, WHICH PERMITS USE, DISTRIBUTION AND REPRODUCTION IN ANY MEDIUM, PROVIDED THE ORIGINAL WORK IS PROPERLY CITED."

\section{RESUMEN}

Se presentan resultados de la investigación básica, sistema de uniones y construcción de prototipo de vivienda rural con Guadua aculeata.

La investigación básica contempla: a partir de la selección y corte, bajo los procedimientos de Montoya, (2006); Cruz Ríos, (2013); Preservado con sales de boro, evaluación de la penetración y retención con los procedimientos de MAVDT, (2010); Posada Giraldo, (2015) y Pérez Cruz, (2018); Secado, bajo los procedimientos de Montoya A. (2006); ISO 22157-1 (2004).

Los ensayos mecánicos en uniones se incluyeron a la Guadua angustifolia, normas NSR-10, ASTM D176 e INECOL (2017), contrastando resultados, se obtuvo contenido de humedad y densidad relativa.

En la segunda fase se construye un prototipo de vivienda rural, el proceso constructivo inicia a partir del acopio del material, acondicionamiento, producción de derivados, trabajos preliminares, sistema estructural, prefabricación de armaduras, colocación, cubierta, entrepiso, muros y acabados.

Se concluye que, los resultados obtenidos de las experimentaciones, permiten obtener modelos de predicción como una herramienta práctica que facilita el trabajo de los usuarios en la construcción de sistemas arquitectónicos. El sistema constructivo comprende desde la selección hasta su aplicación como elemento estructural y recepción de elementos secundarios que conforman las limitantes del espacio, la G. aculeata presenta excelentes condiciones para la producción social del hábitat.

\section{Palabras clave:}

Guadua aculeata, sistema constructivo, hábitat, investigación básica, uniones 


\section{ABSTRACT}

Results of the basic research, union system and prototype construction of rural housing with Guadua aculeata are presented.

The basic research contemplates: from the selection and cutting, under the procedures of Montoya, (2006); Cruz Ríos, (2013); preserved with boron salts, evaluation of penetration and retention with MAVDT procedures, (2010); Posada Giraldo, (2015) and Pérez Cruz, (2018); dried, under the procedures of Montoya A. (2006); ISO 22157-1 (2004).

The mechanical trials in joints were included in the Guadua angustifolia, NSR-10, ASTM D176 and INECOL (2017), contrasting results, moisture content and relative density were obtained.

In the second phase a prototype of rural housing was built, the construction process starts from the collection of the material, conditioning, production of derivatives, preliminary work, structural system, prefabrication of reinforcement, placement, roof, mezzanine, walls and finishes.

It is concluded that, the results obtained from the experiments, allow to obtain prediction models as a practical tool that facilitates the work of users in the construction of architectural systems. The construction system includes from the selection to its application as a structural element and reception of secondary elements that make up the limitations of the space. Guadua aculeata presents excellent conditions for the social production of the habitat.

\section{Key words:}

Guadua aculeata, constructive system, research, basic, prototype.

\section{INTRODUCCIÓN}

En México el bambú $\square$ de la especie Guadua aculeata (tarro), como material de construcción ha sido utilizado desde tiempos prehispánicos, investigaciones arqueológicas demuestran su uso en actividades cotidianas, en la construcción de viviendas en forma rolliza y en reglillas con el sistema de bajareque (INFONAVIT, C. P. (1999). En general los bambúes tienen grandes beneficios ambientales: productor de oxigeno, biomasa, retenedor de $\mathrm{CO} 2$, regenerador de suelos, por su rápido crecimiento útil en la reforestación y para detener la erosión de suelos de márgenes de ríos y arroyos. La Guadua aculeata es un material sustentable con ventajas sobre otros materiales naturales, reemplaza con éxito a la madera y materiales convencionales, su resistencia y menor energía en su obtención lo hacen el material de menor valor en lo referente a su eco-costo (Morán Ubidia, J. A. S/F). Con características como: diámetros de $20 \mathrm{~cm}$, longitud utilizable de $18 \mathrm{~m}$, entrenudos desde $23 \mathrm{~cm}$ hasta $60 \mathrm{~cm}$, espesores de pared de $3 \mathrm{~cm}$ en la parte baja y $7 \mathrm{~mm}$ en la parte alta, rápido crecimiento y propiedades mecánicas importantes.

Las propiedades mecánicas identificadas lo hacen un material apto para construcción Ordoñez Candelaria, V. R., \& Bárcenas Pazos, M. G. (2014); Ordoñez., M. e. (2012); y Zaragoza, Hernández, I. (2012). comparado con otros materiales tiene ventajas de resistencia mecánica. El uso estructural de la Guadua aculeata, se realiza de manera pragmática, el desconocimiento de las condiciones previas al uso del material genera incertidumbre, los riesgos imprevisibles y los daños por el tiempo no pueden ser controlados. La sección cónica, la variabilidad de las secciones, las deformaciones en el eje central longitudinal, dificulta el proceso de ensamblaje de los diferentes elementos de la estructura y no permite la estandarización del proceso de construcción.

El sistema constructivo integra en la primera fase la investigación básica de la Guadua aculeata, como la generación del conocimiento necesario sobre el material, la definición o identificación de las condiciones adecuadas para ser usado como material estructural, a partir de la selección y corte con la medición del contenido de humedad. La 
preservación por medio de soluciones a base de sales de boro por el método de inmersión difusión, su evaluación por penetración y retención en $\mathrm{EAB} / \mathrm{m} 3$ (equivalente de ácido bórico). El secado por métodos naturales a intemperie, en diferentes regiones geográficas y épocas del año.

Los sistemas de unión con pernos, tienen ventajas estructurales comparados con las uniones artesanales, las cuales dificultan el proceso y se prolonga el tiempo de construcción.

Los antecedentes para el estudio del sistema de uniones, están establecidos en Colombianas, Normas Técnicas NTC. (2010). Los valores de diseño para uniones empernadas tipo "P", (tensión paralela a la fibra, "Q" (tensión perpendicular a la fibra) y "T" (compresión perpendicular a la fibra) figura 1.

La segunda fase del sistema constructivo, corresponde a la aplicación de los conocimientos generados en la investigación básica, aplicados en la construcción de un prototipo de vivienda rural, integrando los procedimientos constructivos a partir de la cimentación, anclajes, fijación, relleno de entrenudos con mortero, prefabricación del sistema estructural, sistema de uniones a diferentes esfuerzos mecánicos con pernos de $3 / 8$ plg y $1 / 2$ plg, con mortero y sin mortero, sistema estructural de entrepiso, estructura de cubierta, muros y acabados.

Se presentan los resultados de las experimentaciones realizadas de la investigación básica de la Guadua aculeata: determinación del contenido de humedad al momento del corte por el método gravimétrico en las cuatro fases lunares en época de lluvias y de secas en un año, la evaluación de la solución preservante mediante la medición de la penetración por la aplicación de soluciones reveladoras ante la presencia de las sales de boro, medición de la retención de la solución preservante de sales de boro $E A B / m 3$, por el método de la Azometina $\mathrm{H}$ y espectofotometría, determinación de la velocidad de secado con la medición de la variable contenido de humedad en periodos de tiempo establecidos. Ensayos de tipos de unión, a tensión paralela a la fibra "P", tensión perpendicular a la fibra" Q" y compresión perpendicular a la fibra "T", rellenos con mortero en el entrenudo y sin relleno, con pernos de $3 / 8$ plg y $1 / 2$ plg, de las especies Guadua aculeata y Guadua angustifolia.

Los resultados de la segunda fase; la construcción del prototipo de vivienda rural, desde la extracción de los tallos hasta los acabados interiores. Los tallos utilizados para las experimentaciones de la investigación se colectaron en guaduales de crecimiento natural, la selección, corte y madurez bajo los lineamientos establecidos Morán Ubidia, J. A. (S/F); Cruz Ríos, H. (2013) y Londoño, X. (2005). Los de G. angustifolia de plantaciones en el municipio de Hueytamalco, Puebla y la Guadua aculeata del centro experimental "Las Margaritas" del INIFAP. El material de ensayo se utilizó en condición seca $(\mathrm{CH}<19 \%)$, colombianas, N. T. (2010); Morán Ubidia, J. A. (S/F); Montoya Arango, J. A., \& Jiménez Arias, E. (6 de enero de 2006), NTC 5301, preservado con sales de boro Peña V., L., Burgo s., A., González F., A., \& Will Valero, S. (julio de 2009).

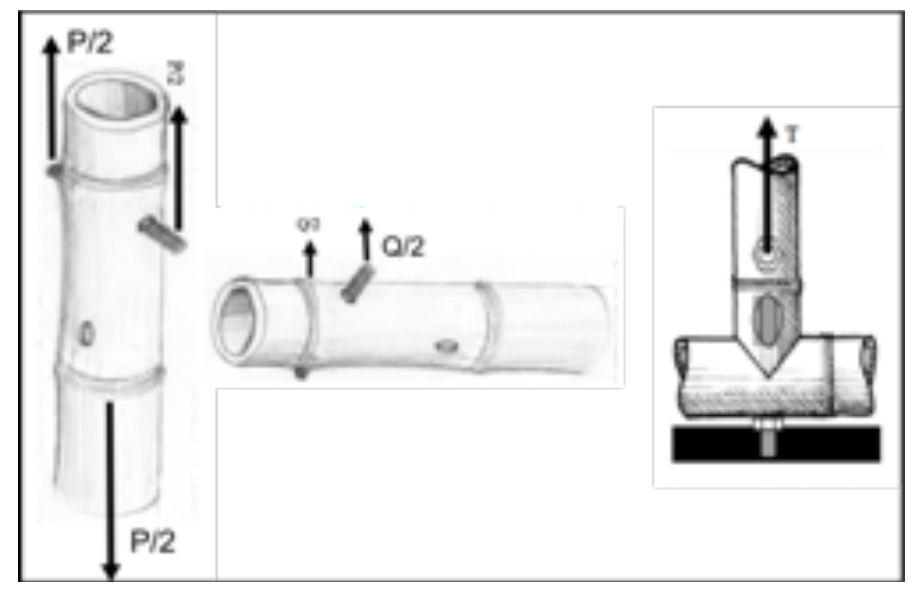

Figura 1. Uniones empernadas "P", "Q" y "T". Tomadas de la Norma Colombiana NSR 10 Capítulo G12 estructuras de Guadua p.G-127. 
Para este estudio el concepto de sistema constructivo se estableció como un conjunto de elementos, técnicas y procedimientos para la construcción de viviendas con Guadua aculeata, que integrados dan orden y sentido funcional.

Las experiencias en la construcción del prototipo de la vivienda rural permitieron establecer los procedimientos constructivos (técnicas) en todas sus etapas, contrastar el cumplimiento de las necesidades funcionales del sistema, las cualidades de los materiales, su producción, vulnerabilidad y la eficiencia del diseño del proceso constructivo.

La prefabricación del sistema estructural, diseñado con elementos que atendieron a exigencias funcionales estructurales con una geometría racional modulada, redujo el tiempo de los procesos constructivos por facilidad del montaje y reducción de mano de obra especializada.

La producción de materiales para la construcción del prototipo es producto de las experimentaciones del proceso de acondicionamiento de la Guadua aculeata para uso estructural.

La construcción del prototipo de la vivienda representa la etapa final de la investigación realizada, concentra los resultados de las experimentaciones con aculeata para determinar las condiciones previas al uso.

\section{METODOLOGÍA}

\subsection{LA INVESTIGACIÓN BÁSICA.}

\subsubsection{SELECCIÓN Y CORTE.}

Comprende la generación del conocimiento necesario sobre el material, para ser usado como material estructural. Todas las muestras fueron extraídas en tres guaduales distintos, identificadas de manera inequívoca "recias" o "maduras" según criterio de observación Morán Ubidia, (s/f); Cruz Ríos, (2013). Los tallos se cortaron en el sitio durante cada fase lunar. Se realizaron dos cortes en dos periodos lunares, se cortaron dos rodajas de $5 \mathrm{~cm}$ de altura de las partes baja, media y alta de 6 tallos extraídos, con un metro de separación entre corte y corte, 36 muestras por fase lunar, con un total de 360 muestras en los dos periodos. Se midió el Chi (contenido de humedad inicial) a cada muestra por el método gravimétrico (Montoya, 2006). Se pesaron para obtener su peso inicial (PI), posteriormente se secaron a temperatura ambiente bajo sombra, se introdujeron al horno de secado durante 48 horas a temperatura $103^{\circ} \mathrm{C} \pm 2^{\circ} \mathrm{C}$, obteniendo su peso anhidro, $(0 \% \mathrm{CH})$. Con los valores obtenidos, se determinó el Chi en porcentaje.

\subsubsection{LA PRESERVACIÓN.}

Se utilizó el método de inmersión-difusión, una solución química hidrosoluble, a base sales de boro como tratamiento para prevenir o contrarrestar la acción de alguno o varios tipos de organismos que destruyen o afectan la integridad de la guadua, las proporciones corresponden a MAVDT, (2010); ICONTEC, (2006); y NTC, (5301). Es incolora e inodora, de baja toxicidad para los mamíferos, efectiva y económica, mientras no esté expuesta a altas humedades y se encuentre protegida por los rayos del sol (Turner, 2008) y (Peña y González, 2009).

El diseño experimental fue de tres niveles de altura en el tallo (baja, media y alta); tres niveles de concentración de la solución preservadora ( $4 \%, 5 \%$ y $6 \%$ ); y tres periodos $(4,5$, y 6 días) con tres repeticiones por nivel, en total 81 muestras de $1.5 \mathrm{~m}$ de longitud, de las cuales se obtuvieron las rodajas de $5 \mathrm{~cm}$ de altura, 81 muestras para la penetración, 81 muestras para retención, 81 muestras para la densidad y 81 para $\mathrm{CH}$. Una vez preservadas se colocaron a la intemperie para secarlas por medios naturales, hasta bajar el $\mathrm{CH}$ a menos del $19 \%$.

La evaluación de la penetración de la solución preservante con sales de boro, fue con el método de (Erdoiza y Echenique, 1980), con indicadores de la retención de EAB alcanzada en el material preservado: un rojo brillante 
puede contener $\geq 1.28 \mathrm{~kg} / \mathrm{m} 3$, el rojo marrón a amarillo marrón puede contener @ 0.96kg/m3, y el amarillo @ 0.32 $\mathrm{kg} / \mathrm{m} 3$ (FAO, 1986).

Para determinar la retención de las SB en $\mathrm{kg} / \mathrm{m} 3 \mathrm{EAB}$, se aplicó un método cuantitativo, espectrofotométrico para boro (B) mediante la formación de un complejo coloreado con Azometina $\mathrm{H}$ de acuerdo con lo descrito por Pérez Cruz, (2018) y Ponce, D. L. (2010).

\subsubsection{PROCESO DE SECADO.}

La experimentación se realizó en dos épocas; de lluvias y secas y dos ambientes geográficos, la selección de las muestras por el mismo procedimiento para todas las experimentaciones, las muestras con una longitud de $6 \mathrm{~m}$ y diámetros diversos, se realizaron cortes trasversales en rodajas de $5 \mathrm{~cm}$ de altura en las partes baja y alta de cada tallo en diferentes periodos, establecidos de acuerdo a la norma ISO 22157-1 (2004), numeral 6 y la norma DIN 52183 (citada en Montoya Arango \& Giménez Arias, 2006. Se midió el contenido de humedad por el método gravimétrico registrando los valores obtenidos hasta lograr reducir hasta menos del $18 \%$ para ser usado como material estructural (GOCDMX, NTC 2017).

\subsubsection{SISTEMA DE UNIONES.}

Se prepararon muestras con la calidad establecida en las normas, longitud de un entrenudo más $20 \mathrm{~cm}$ de cada extremo. Los ensayos fueron realizados con los procedimientos de la norma colombiana, título G, NSR-10, capítulo G12: distancia al nudo $50 \mathrm{~mm}$, distancias mínimas del perno al extremo y dimensiones de arandelas. Complementándose con la norma ASTM D1761: la distancia entre las reacciones es $300 \mathrm{~mm}$, superando los tres diámetros en la mayoría de los ensayos. Velocidad de carga $(0,9 \mathrm{~mm} / \mathrm{min})$, en una máquina universal de pruebas. Se realizaron 144 ensayos, 108 con Guadua aculeata y 36 con Guadua angustifolia; Tensión paralela a la fibra "P", tensión perpendicular a la fibra "Q" y compresión perpendicular a la fibra "T". 72 ensayos tipo "P" "Q" y "T" con mortero y $\sin$ mortero con $G$. aculeata con perno de 3/8", colocados a $5 \mathrm{~cm}$ del nudo, 18 con mortero con perno de 12 ", 18 sin mortero con perno de 12 ". 36 ensayos con G. angustifolia tipo "P" "Q" y "T" con y sin mortero con perno de 12 " colocados a $5 \mathrm{~cm}$ del nudo.

\subsubsection{SISTEMA CONSTRUCTIVO.}

La construcción del prototipo representa la parte final de la investigación e integra los resultados de las experimentaciones de la investigación básica y los resultados del proceso constructivo en sus diferentes etapas. La producción del material, selección y corte, con la metodología generada producto de las experimentaciones realizadas, con las condiciones de madurez según Morán Ubidia, (s/f) y Cruz Ríos, (2013). El preservado se realizó por el método de difusión-inmersión con sales de boro al $6 \%$ y durante 6 días, derivado de las experimentaciones realizadas y la NTC (5301); Peña, (2009). El secado bajo sombra y a la intemperie se realizó una vez que fueron extraídos de la pileta de preservación, colocados verticalmente para el escurrimiento del exceso de preservante, se rotaban cada 8 días, se pesaban y media el $\mathrm{CH}$ en periodos de tiempo establecidos (Montoya, 2006).

La prefabricación de armaduras, con uniones a tensión paralela a la fibra y tensión perpendicular a la fibra derivadas de las experimentaciones en el sistema de uniones, el trazo y armado en el sitio con sistemas geométricos básicos, las uniones con pernos de $3 / 8$ plg y $1 / 2$ plg, con mortero y sin mortero. La construcción del sistema estructural inicia con el izado y colocación de las armaduras en cada eje estructural, colocación de postes, vigas longitudinales entre armaduras, riostras diagonales entre postes y entre armaduras, elementos de unión entre armaduras en la cubierta, las uniones se estabilizan dependiendo del esfuerzo actuante, con pernos y rellenos de mortero. La construcción del entrepiso, cubierta y muros, así como el anclaje con los procedimientos establecidos en (MAVDT, 2010). La producción de reglilla y esterilla, fue determinada por las experimentaciones realizadas previamente, posteriormente preservadas y secadas según Turner, (2008); Peña y González, (2009) y Montoya, (2006). 


\section{RESULTADOS}

\subsection{SELECCIÓN Y CORTE.}

Los contenidos de humedad obtenidos por fase lunar presentaron una gran variabilidad en los dos periodos de lluvias y de secas, la diferencia entre los promedios de cuarto menguante es de $46.3 \%$, en luna nueva es de 6.9 $\%$, en cuarto creciente es de $23 \%$, en luna llena es de $25.2 \%$. El segundo registro de cuarto menguantes fue de $5.6 \%$. En la época de lluvias el menor contenido de humedad se obtuvo en el cuarto menguante y luna llena, en la época de secas en luna llena y cuarto creciente Tabla 1.

Tabla 1.

Resumen de resultados promedio de contenido de humedad inicial de muestras de $G$. aculeata de la región de Hueytamalco, Puebla, por fase lunar, periodos 2017-2018. Fuente: elaboración propia.

\begin{tabular}{|c|c|c|c|c|c|c|c|}
\hline Ubicación & Periodo & Clave & $\begin{array}{c}\text { Cuarto } \\
\text { menguante }\end{array}$ & $\begin{array}{l}\text { Luna } \\
\text { nueva }\end{array}$ & $\begin{array}{l}\text { Cuarto } \\
\text { creciente }\end{array}$ & $\begin{array}{l}\text { Luna } \\
\text { llena }\end{array}$ & $\begin{array}{c}\text { Cuarto } \\
\text { menguante }\end{array}$ \\
\hline \multirow{3}{*}{ Guadual 2} & \multirow{6}{*}{$\begin{array}{l}\text { Mayo-junio } \\
2017\end{array}$} & 1 & 116.5 & 90.5 & 108.2 & 105.8 & 94.6 \\
\hline & & 2 & 89.2 & 159.9 & 110.6 & 102.0 & 108.5 \\
\hline & & 3 & 103.3 & 121.4 & 97.6 & 102.1 & 109.3 \\
\hline \multirow{3}{*}{ Guadual1 } & & 4 & 91.8 & 83.9 & 95.3 & 90.6 & 112.9 \\
\hline & & 5 & 101.8 & 101.5 & 100.3 & 87.5 & 94.8 \\
\hline & & 6 & 78.8 & 92.2 & 110.6 & 93.7 & 115.6 \\
\hline \multicolumn{3}{|c|}{ Promedio } & 96.9 & 108.2 & 103.8 & 96.9 & 106.0 \\
\hline \multirow{6}{*}{ Palmatita } & \multirow{6}{*}{$\begin{array}{c}\text { Febrero- } \\
\text { marzo } 2018\end{array}$} & 1 & 117.2 & 128.3 & 61.8 & 55.1 & 102.1 \\
\hline & & 2 & 185.7 & 159.4 & 79.2 & 92.0 & 144.6 \\
\hline & & 3 & 130.0 & 78.1 & 67.5 & 83.6 & 90.4 \\
\hline & & 4 & 127.1 & 112.8 & 67.2 & 78.1 & 84.0 \\
\hline & & 5 & 134.9 & 104.5 & 102.1 & 63.8 & 86.6 \\
\hline & & 6 & 164.7 & 108.0 & 105.8 & 57.5 & 94.1 \\
\hline \multicolumn{3}{|c|}{ Promedio } & 143.3 & 115.2 & 80.6 & 71.7 & 100.3 \\
\hline
\end{tabular}

\subsection{EVALUACIÓN DE LA PRESERVACIÓN.}

En general se observa que la penetración fue en todo el espesor de las muestras, la parte interna del tallo se tornó a un color rojo brillante, lo que indica que existe una mayor concentración de sales de boro, sobre todo en las muestras de la parte baja de los tallos, se puede explicar porque en la parte interna de la pared de los tallos, hay una menor cantidad de fibras, esta cantidad aumenta hacia al exterior del tallo. En varias muestras se observa un color café rojizo, en otras se observa en la parte próxima a la externa un color café amarillento figura 2.

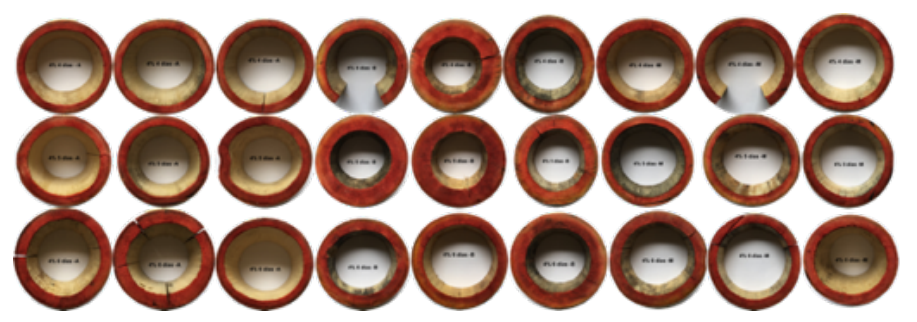

Figura 2. Resultados de la evaluación penetración y presencia de SB, al $4 \%$, en 4,5 y 6 días en las partes baja, media y alta del tallo, preservadas con SB por inmersión-difusión. G. aculeata de la región de Hueytamalco, Puebla.
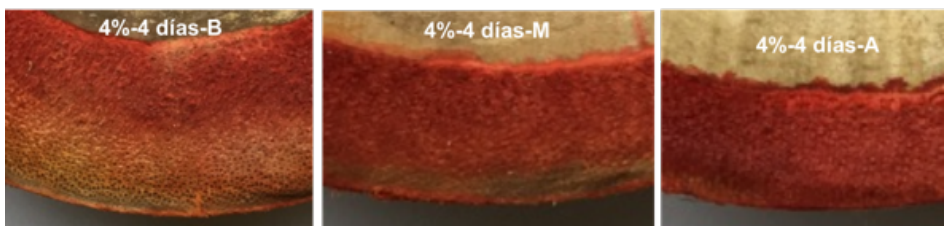

Figura 3. Resultados, detalle de penetración-presencia de SB, concentración al $4 \%$ en 4 días, partes baja, media y alta del tallo, preservadas por inmersión-difusión. G. aculeata de la región de Hueytamalco, Puebla. 
En resumen, del total de las 81 muestras se identificaron siete muestras que representan el $9 \%$, que alcanzaron penetración irregular café amarillento PTIA, con un estimado de $0.20 \% \mathrm{EAB}$, las once que representan el $14 \%$, con penetración total regular PTR rojo brillante, con un estimado de .30 \% EAB y las 63 que representan el $77 \%$ con penetración total irregular PTI café rojizo con un estimado de $0.25 \%$ EAB, por encima de lo que establece la (FAO, 1986) figura 3.

La retención de las SB es equivalente a la absorción neta que se expresa en $\mathrm{kg} / \mathrm{m} 3$. Los resultados promedio obtenidos se presentan en la Tabla 2 separados por niveles: altura del tallo, concentración de la solución preservadora y periodos de inmersión, se consideran dos resultados el primero con un g de material analizado EAB y el segundo el mismo material escalado al volumen del material tratado con la incorporación de la densidad básica $(\mathrm{DB}), \mathrm{EAB}$.

Por periodos de inmersión el mayor promedio obtenido de retención de SB fue a los 6 días con $3.01 \mathrm{~kg} / \mathrm{m} 3$, a los 4 días con $2.88 \mathrm{~kg} / \mathrm{m} 3$ y finalmente a los 5 días con $2.24 \mathrm{~kg} / \mathrm{m} 3$. Los resultados obtenidos son superiores a lo que establece la (FAO, 1986).

Tabla 2.

Retención en las muestras de sales de boro en $\mathrm{kg} / \mathrm{m} 3 \mathrm{EAB}$, en las partes del tallo (baja, media y alta), concentraciones de sales de boro en la solución preservadora (4 \%,5 \% y $6 \%$ ), y tres periodos de inmersión $(4,5$ y 6 días), G. aculeata de la región de Hueytamalco, Puebla.

EAB* cálculos obtenidos por (Cruz Pérez, 2018), EAB** cálculos obtenidos por el autor.

\begin{tabular}{|c|c|c|c|c|c|c|c|}
\hline \multirow[t]{2}{*}{$\begin{array}{l}\text { Parte del } \\
\text { tallo }\end{array}$} & $\begin{array}{l}\text { Concentración } \\
\text { de la solución }\end{array}$ & Inmersión & $\mathrm{EAB}^{*}$ & $\begin{array}{c}\text { Concentración } \\
\text { de B }\end{array}$ & B & $\begin{array}{c}\text { Densidad } \\
\text { básica (DB) }\end{array}$ & $\mathrm{EAB}^{* *}$ \\
\hline & (\% de SB) & Días & $\mathrm{kg} / \mathrm{m} 3$ & $\mathrm{mg} / \mathrm{kg}$ & $\mathbf{k g} / \mathbf{k g}$ & $\mathrm{kg} / \mathrm{m} 3$ & $\mathrm{~kg} / \mathrm{m} 3$ \\
\hline \multirow[t]{9}{*}{ Baja } & \multirow[t]{3}{*}{4} & 4 & 2.20 & 2200 & 0.0022 & 522 & 1.15 \\
\hline & & 5 & 1.00 & 1000 & 0.0010 & 490 & 0.49 \\
\hline & & 6 & 5.10 & 5105 & 0.0051 & 509 & 2.60 \\
\hline & \multirow[t]{3}{*}{5} & 4 & 2.03 & 2030 & 0.0020 & 510 & 1.04 \\
\hline & & 5 & 1.40 & 1400 & 0.0014 & 478 & 0.67 \\
\hline & & 6 & 1.10 & 1104 & 0.0011 & 509 & 0.56 \\
\hline & \multirow[t]{3}{*}{6} & 4 & 4.24 & 4241 & 0.0042 & 546 & 2.32 \\
\hline & & 5 & 3.21 & 3210 & 0.0032 & 564 & 1.81 \\
\hline & & 6 & 1.08 & 1080 & 0.0011 & 544 & 0.59 \\
\hline \multirow[t]{9}{*}{ Media } & \multirow[t]{3}{*}{4} & 4 & 1.31 & 1310 & 0.0013 & 558 & 0.73 \\
\hline & & 5 & 2.01 & 2010 & 0.0020 & 600 & 1.21 \\
\hline & & 6 & 2.02 & 2015 & 0.0020 & 611 & 1.23 \\
\hline & \multirow[t]{3}{*}{5} & 4 & 6.52 & 4410 & 0.0044 & 583 & 2.57 \\
\hline & & 5 & 4.41 & 3300 & 0.0033 & 618 & 2.04 \\
\hline & & 6 & 6.63 & 6636 & 0.0066 & 528 & 3.51 \\
\hline & \multirow[t]{3}{*}{6} & 4 & 1.70 & 1701 & 0.0017 & 598 & 1.02 \\
\hline & & 5 & 1.12 & 1120 & 0.0011 & 589 & 0.66 \\
\hline & & 6 & 2.21 & 2212 & 0.0022 & 591 & 1.31 \\
\hline \multirow[t]{9}{*}{ Alta } & \multirow[t]{3}{*}{4} & 4 & 2.10 & 2100 & 0.0021 & 594 & 1.25 \\
\hline & & 5 & 1.46 & 1460 & 0.0015 & 657 & 0.96 \\
\hline & & 6 & 3.15 & 3150 & 0.0032 & 589 & 1.86 \\
\hline & \multirow[t]{3}{*}{5} & 4 & 3.30 & 3300 & 0.0033 & 607 & 2.00 \\
\hline & & 5 & 4.56 & 4565 & 0.0046 & 644 & 2.94 \\
\hline & & 6 & 4.67 & 4670 & 0.0047 & 589 & 2.75 \\
\hline & \multirow[t]{3}{*}{6} & 4 & 2.28 & 2250 & 0.0023 & 689 & 1.55 \\
\hline & & 5 & 1.00 & 1005 & 0.0010 & 610 & 0.61 \\
\hline & & 6 & 1.10 & 1105 & 0.0011 & 616 & 0.68 \\
\hline
\end{tabular}

Los resultados promedio con relación a la altura del tallo, son contrarios al supuesto de mayor densidad menor retención. A mayor concentración de SB, mayor retención y con mayor tiempo de inmersión mayor retención.

\subsection{EL SECADO POR MEDIOS NATURALES A INTEMPERIE.}

Las experimentaciones realizadas en la ciudad de Xalapa, arrojaron el tiempo de secado para el periodo octubre 2016-febrero 2017 el promedio de contenido de humedad obtenido en la primera medición fue de $71.1 \%$, a los 21 días de $45.3 \%$, a los 42 días de $27.1 \%$, a los 62 días de 19.3\%, a los 103 días de $19.1 \%$ y a los 125 días fue de $16.5 \%$ de $\mathrm{CH}$. En la ciudad de Papantla, en el periodo septiembre 2016-enero 2017, en la primera medición se obtuvieron $\mathrm{CH}$ en promedio de $75.1 \%$, a los 21 días de $39 \%$, a los 40 días de $13.2 \%$, a los 64 días de 19.6\%, 
a los 84 días de $17.1 \%$ a los 105 días de $15.6 \%$ y a los 132 días de $12.2 \%$. En contraste durante el secado en el periodo de marzo a mayo en Papantla en Chi fue de 71\% y en 56 días alcanzó un CHE de 16.88 \%. Con estos resultados se denota claramente el efecto de la temperatura y la humedad sobre el secado al aire libre, puesto que entre septiembre y febrero es más frecuente la precipitación pluvial y más baja la temperatura, mientras que entre marzo y mayo es escasa la precipitación pluvial, con mayor número de días no nublados y empieza a elevarse la temperatura.

En la figura 4 se presenta un modelo de predicción para calcular el CH al 19\% establecido en GOCDMX, NTC. (2017). en la ciudad de Xalapa se identifica a los 64 días, en Papantla en el mismo periodo a los 56 días, en Papantla en el periodo marzo-mayo a los 28 días.

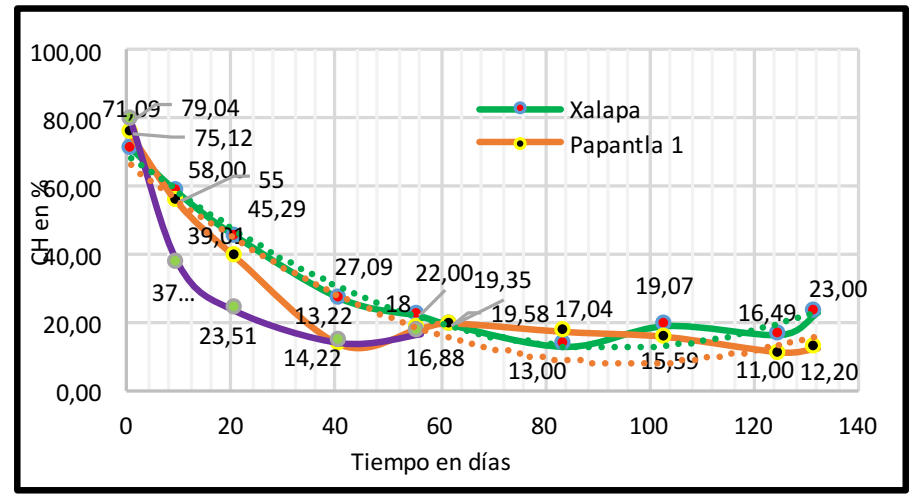

Figura 4. Comparación entre los tres procesos de secado de tallos de G. aculeata de la región de Hueytamalco, Pue. Xalapa octubre 2016 febrero 2017 y Papantla, Ver., septiembre 2016 febrero 2017 y marzo-mayo 2017.

\subsection{SISTEMA DE UNIONES.}

Los resultados en el sistema de uniones con G. angustifolia sin mortero y perno de $1 / 2$ " registran un incremento respecto a las uniones con G. aculeata con las mismas características. $77 \%$ para tensión paralela $(P), 79.6 \%$ para compresión perpendicular (T) y 39.6\% para tensión perpendicular a la fibra (Q). Las cargas máximas registradas en uniones tipo "P", "Q" y "T" sin mortero y perno de 12", en G. aculeata, comparadas con la G. angustifolia, denotan una diferencia importante, la cual se encuentra relacionada con el incremento del diámetro y el espesor de pared. El 4\%, 10\% y 12\% para el diámetro y 37\%, 33\% y 38\% para el espesor de pared, figura 5 . Con mortero y perno de 12 " el incremento para tensión paralela $(\mathrm{P})$ es de $7.6 \%$, en compresión perpendicular (T) la G. aculeata supera ligeramente a la G. angustifolia, en tensión perpendicular (Q) las uniones con G. aculeata superan a las con G. angustifolia en $17.3 \%$, figura 6 .

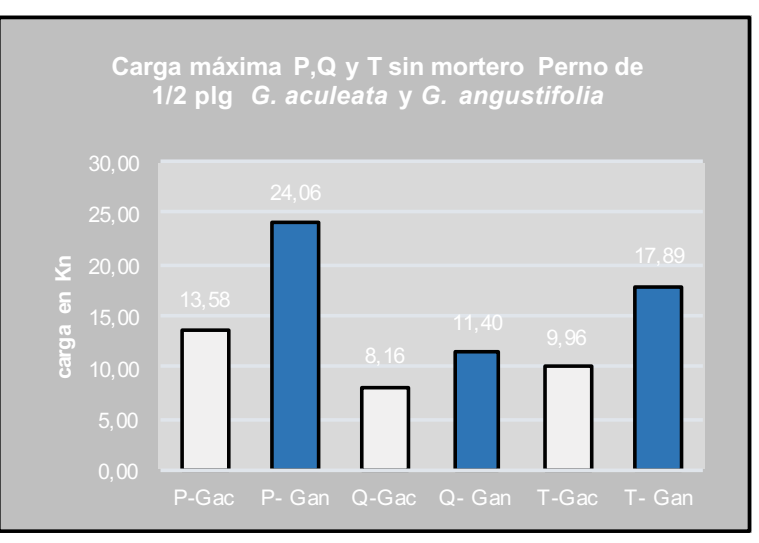

Figura 5. Resultados uniones "P", "Q" y "T" G. aculeata y G. angustifolia, perno de 12 ", sin mortero, carga máxima.

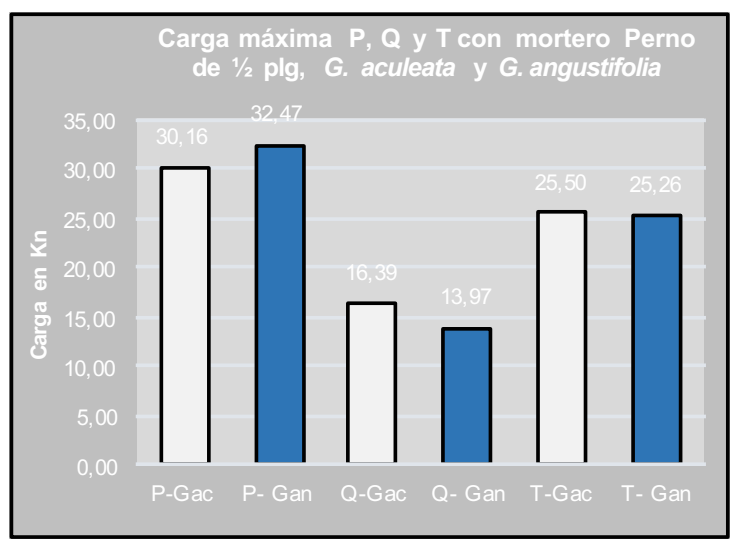

Figura 6. Resultados uniones "P", "Q" y "T" G. aculeata y G. angustifolia, perno de 12 ", con mortero, carga máxima 
Con mortero y perno de 12 " el incremento para tensión paralela $(P)$ es de $7.6 \%$, en compresión perpendicular $(T)$ la $G$. aculeata supera ligeramente a la $G$. angustifolia, en tensión perpendicular (Q) las uniones con $G$. aculeata superan a las con G. angustifolia en $17.3 \%$, figura 6 .

\subsection{CONSTRUCCIÓN DEL PROTOTIPO.}

Los resultados de la construcción del prototipo en sus diferentes etapas, formulan procedimientos y técnicas constructivas para integrarlas al sistema constructivo.

Los elementos que integran el sistema constructivo inician desde la producción del material, trabajos preliminares, prefabricación de elementos estructurales; armaduras, vigas compuestas, cuerdas inferiores y superiores de la armadura, construcción del prototipo; armado de elementos prefabricados, construcción de cubierta, entrepiso, muros y evaluación del proceso constructivo.

Corte y selección: se extrajeron 450 tallos de 6 m de longitud de diámetros diferentes, 200 para el sistema estructural y 250 para la producción de esterilla y reglilla figura 7 .

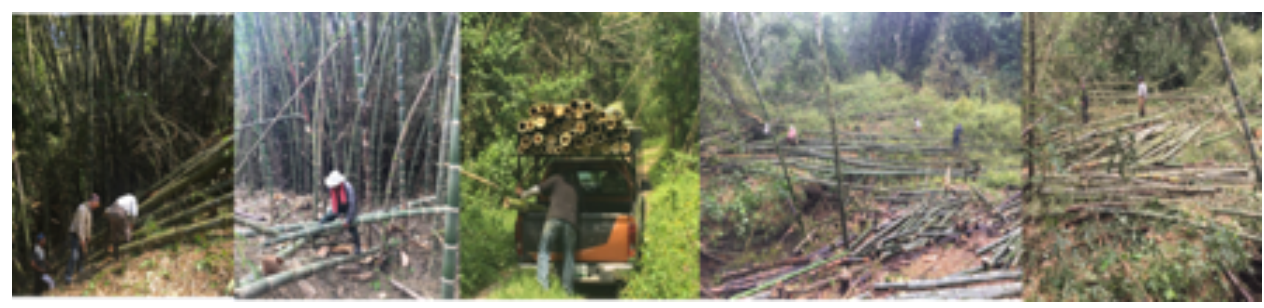

Figura 7. Extracción de tallos, corte, selección, clasificación y transportación a sitio para acondicionamiento.

La preservación de los tallos se realizó con el procedimiento experimentado, con sales de boro, por inmersióndifusión figura 8.

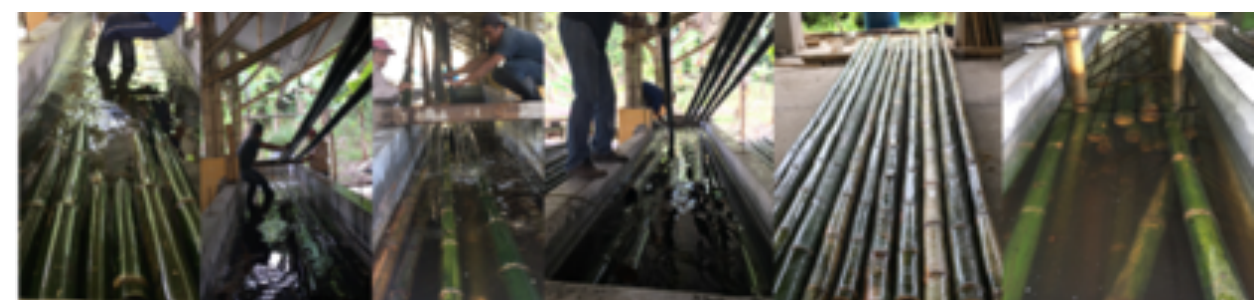

Figura 8. Proceso de preservado por inmersión-difusión, con solución a base de sales de boro.

El secado fue bajo sombra por las altas temperaturas y humedad relativa presentadas en el sitio, el proceso de secado requiere un control y medición del contenido de humedad. Se tomaron mediciones a algunas muestras durante el proceso de secado de manera aleatoria, bajo los procedimientos de la metodología empleada por (Montoya, 2006) figura 9.

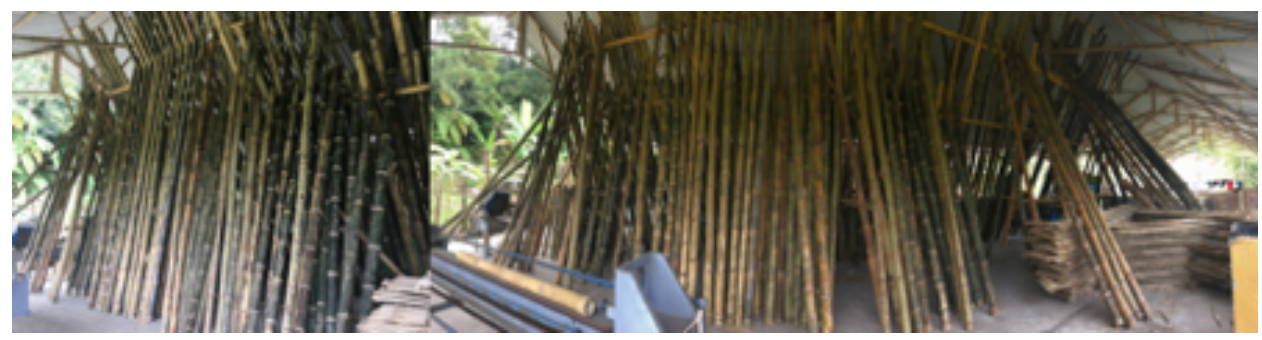

Figura 9. Proceso de secado bajo sombra tallos rollizos, esterilla y reglilla.

Los derivados de los tallos de la parte media, esterilla y reglilla, se obtuvieron de manera artesanal con herramientas manuales, para muros, entrepiso y plafón de la cubierta. Fueron preservados y secados figura 10. 

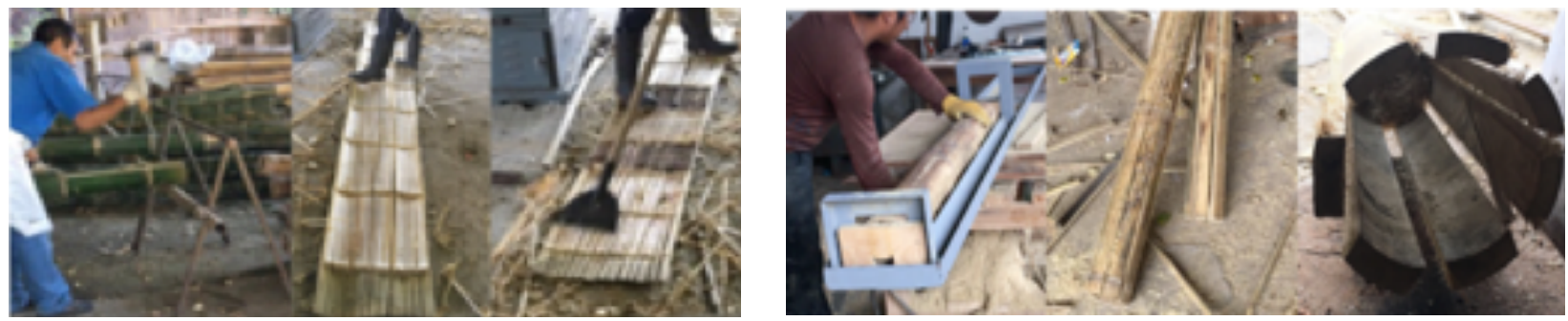

Figura 10. Producción de esterilla y reglilla por medios artesanales.

El sistema estructural fue anclado a la cimentación con varillas de 3/8" y pernos de $3 / 8 \mathrm{plg}$ y $1 / 2 \mathrm{plg}$, con los entrenudos rellenos con mortero, el sistema de cimentación funciona a la inversa que el sistema tradicional constructivo, por su bajo peso $(1.8 \mathrm{~kg} / \mathrm{ml})$ tiene que estar anclado, ante ráfagas de viento imprevistos. Los muros divisorios se anclaron a la trabe de liga, a la cadena de desplante y al piso, según los desempeños de cada elemento figura 11.
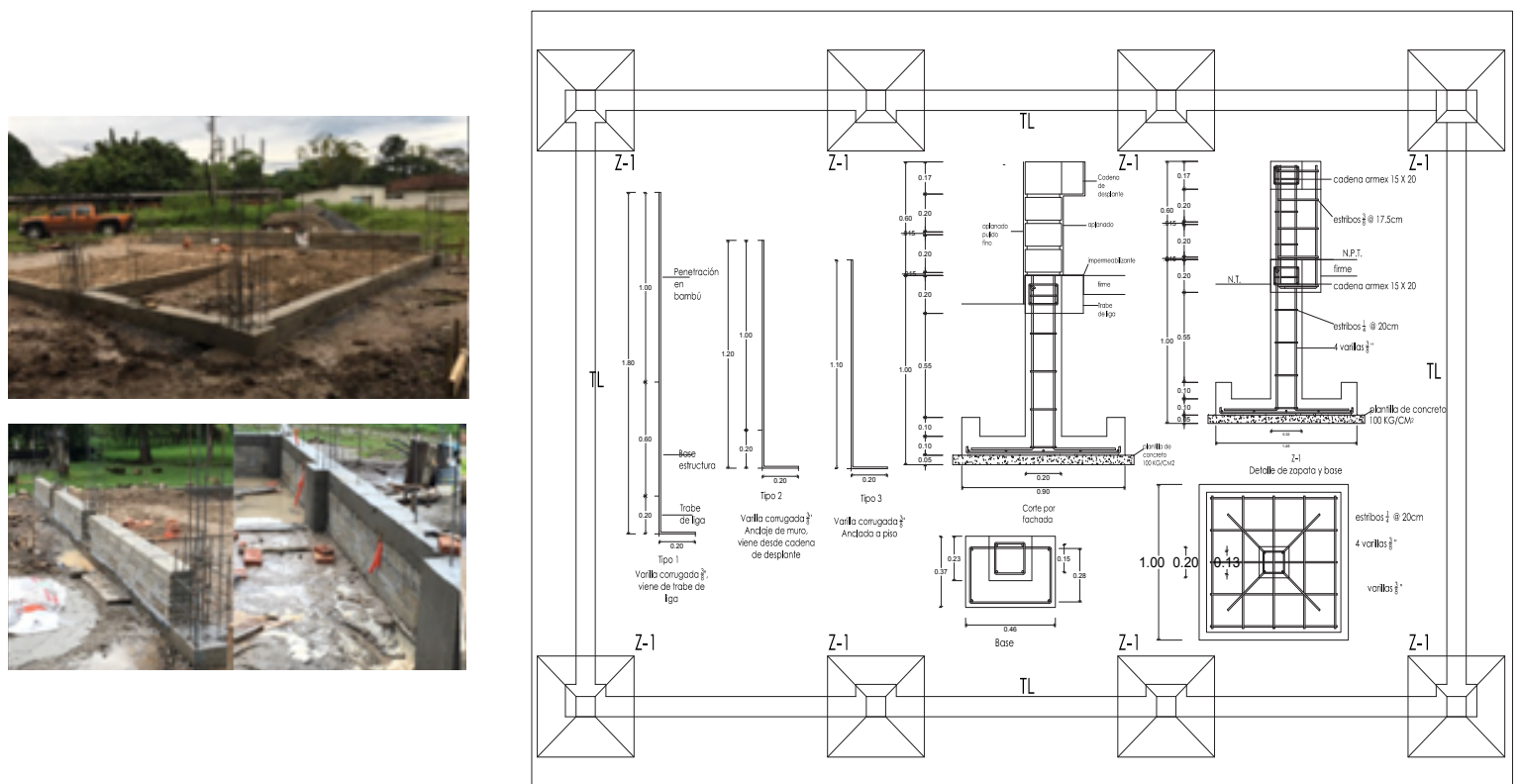

Figura 11. Proceso constructivo cimentación, armados y tipos de anclaje.

El análisis estructural determinó el uso de vigas compuestas y riostras. El procedimiento constructivo se realizó partiendo de la técnica para la selección del material, sin deformaciones del eje central longitudinal, diámetro, conicidad, calidad. Las uniones se realizaron con varilla roscada de $3 / 8 \mathrm{plg}$ con tuercas y arandelas resultado de las experimentaciones realizadas figura 12 .

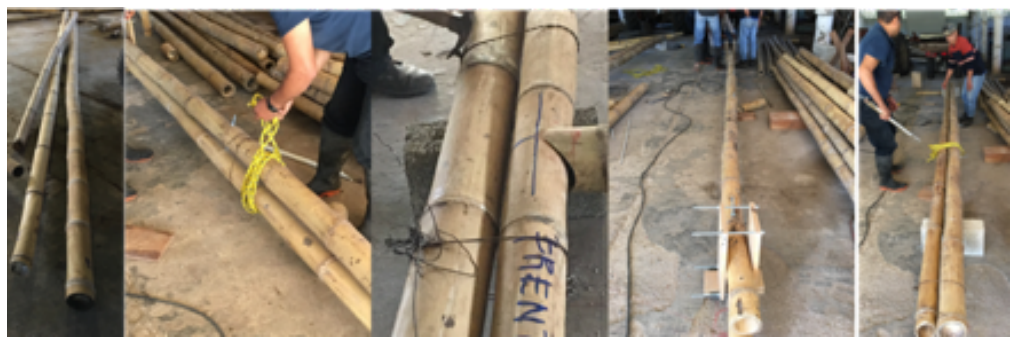

Figura 12. Amarres provisionales, sujeción de elementos para su fijación con varilla roscada.

La configuración del sistema estructural está compuesta por armaduras porticadas moduladas, sus dimensiones y proporciones son originadas de la subdivisión del cuadrado, logrando uniformidad en los ángulos, uniones a boca 
de pescado y pico de flauta, colocadas en plano seriado facilitan la coordinación de los elementos estructurales como postes y vigas y la modulación de elementos de cerramiento, muros perimetrales, interiores y acabados figura 13.
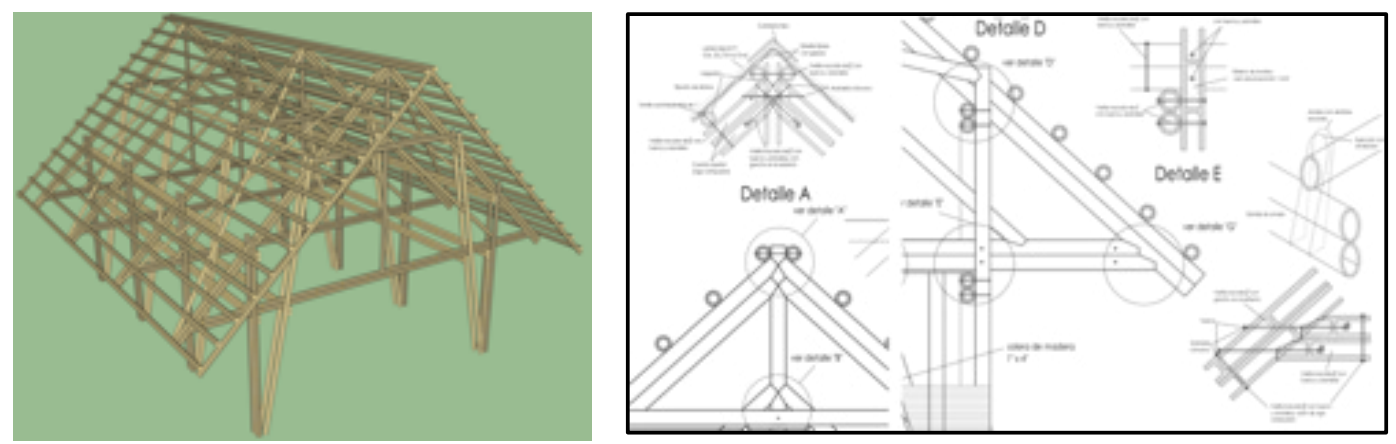

Figura 13. Detalles tipos de uniones en armadura.

El procedimiento constructivo de las armaduras porticadas una vez seleccionado el material, amarres provisionales con cuerda y alambre recocido, cortes a boca de pescado y pico de flauta, perforando el diafragma para introducir la varilla roscada figura 14 .
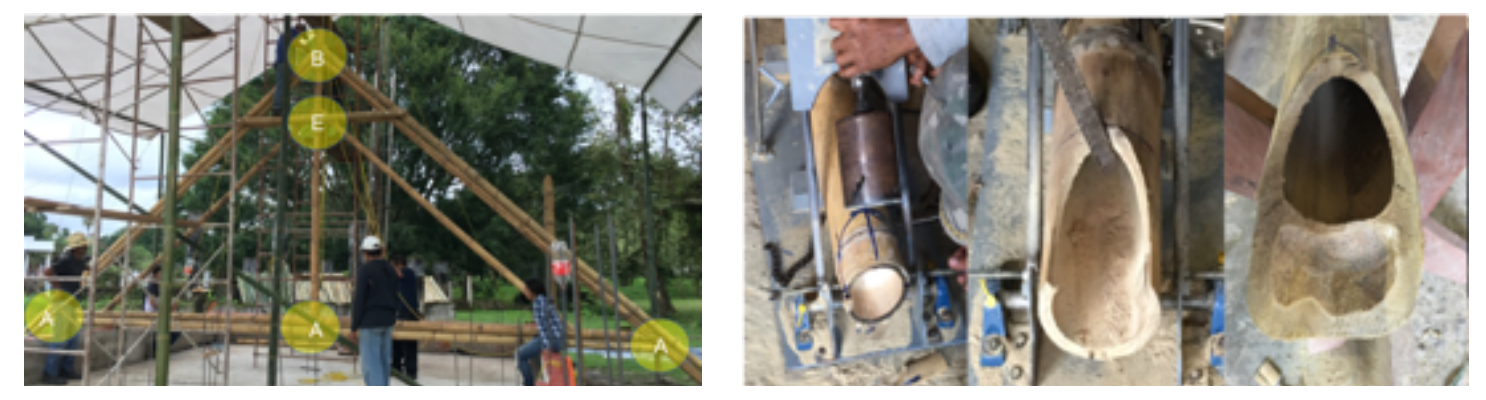

Figura 14. Armadura, uniones y elementos componentes. Tipos de cortes, utilizados en las uniones.

La construcción se realiza en cuatro etapas, en la primera se construye el sistema estructural, integrando la colocación de armaduras, postes verticales, diagonales, y elementos rigidizantes, riostras longitudinales y diagonales, largueros de cubierta, estructura del tapanco, fijación de esterilla y relleno de entrenudos con mortero en uniones ensayadas figura 15.
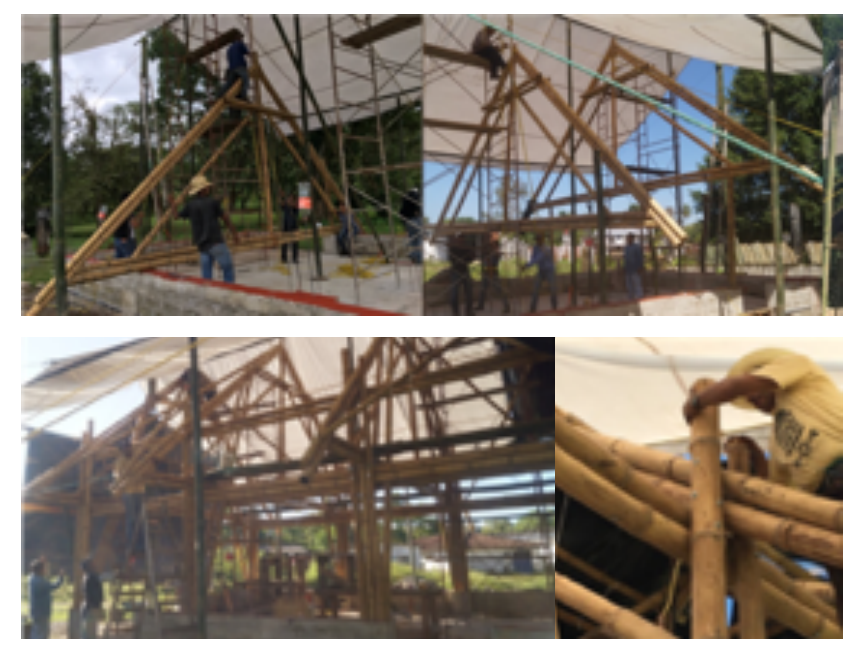

Figura 15. Rigidización de las armaduras, colocación de riostras longitudinales. 
Las uniones en el sistema estructural fueron a tensión paralela a la fibra, tensión perpendicular a la fibra y compresión perpendicular a la fibra, unidas con pernos de 3/8plg y 1/2plg, rellenos de mortero figura 16 .

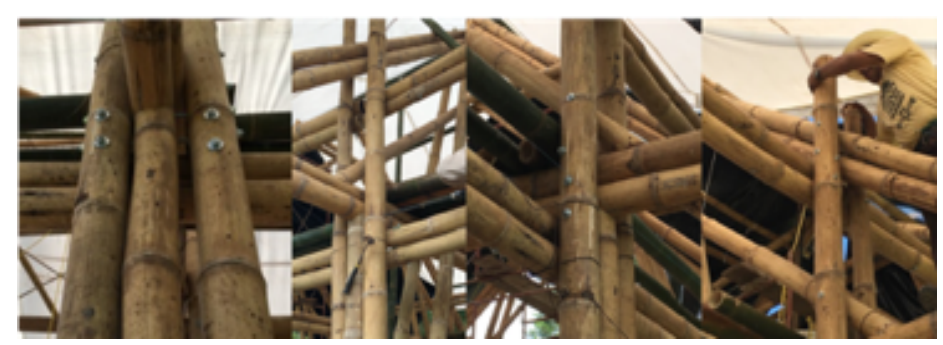

Figura 16. Uniones poste vertical con cuerdas inferiores, riostras longitudinales y cuerdas superiores.

La segunda contempló el armado de muros, incluye: los muros perimetrales, interiores y llenado de entrenudos con mortero. La tercera fase contempló acabados en muros, interiores y exteriores, colocación de reglilla y esterilla, malla gallinero, aplanados con mortero y aplicación de pintura, en interiores de cocina colocación de esterilla, malla gallinero y aplanados con mortero, en plafón de cubierta, colocación de esterilla en la parte baja de la estructura figura 17.

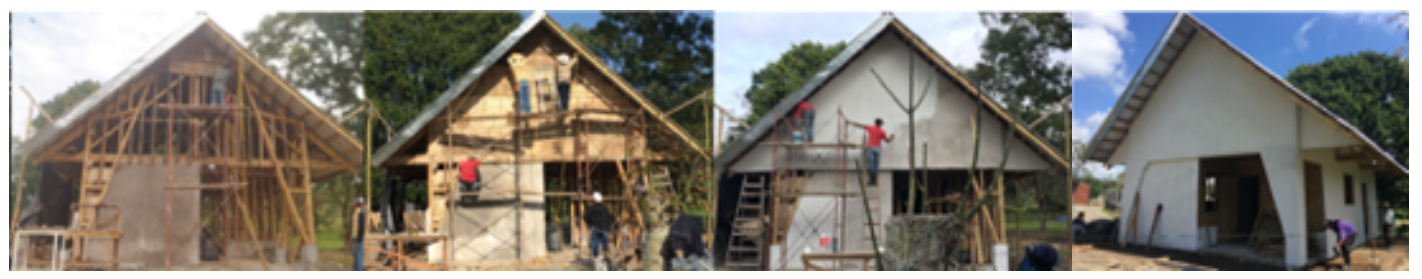

Figura 17. Estructura de muros, recubrimiento con esterilla y aplanado.

La cuarta fase contempló la carpintería, incluye: la construcción de puertas y ventanas con reglilla, colocación de reglilla en muros interiores figura 18.

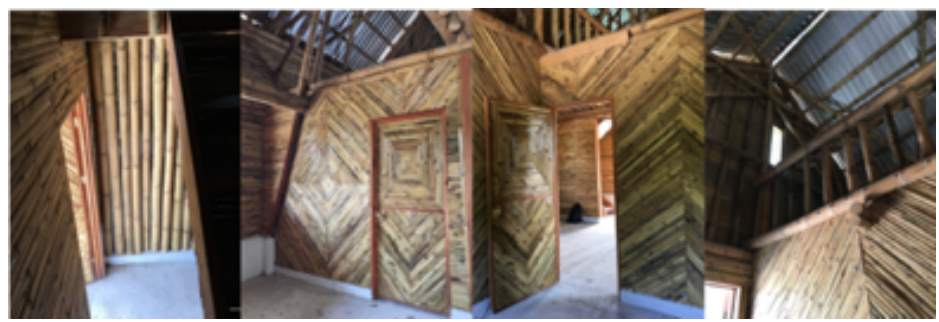

Figura 18. Acabados muros interiores, reglilla con barniz.

\section{CONCLUSIONES}

El corte en época de lluvias en fase lunar menguante presenta menores contenidos de humedad inicial a lo largo del tallo, en época de secas la fase lunar llena y creciente presenta menores contenidos de humedad. Este estudio no es concluyente, es necesario continuar con más experimentaciones a lo largo del año y comparar resultados con otros estudios en las mismas condiciones.

La evaluación de la penetración de las sales de boro en general la mayor parte de las muestras presentaron resultados similares a los encontrados por (Morales Pinzón, 2006), una presencia casi total, observando una zona con penetración o presencia alta, donde el color rojo presentó una tonalidad mayor, corresponde a la parte interna del tallo, lo anterior se explica porque en la pared interior del bambú existe una mayor porosidad y menor densidad, hacia el exterior del bambú disminuye la porosidad y aumenta la densidad, lo que hace difícil la penetración del preservante. El mismo efecto se presentó en la parte baja del tallo, donde se obtuvieron mayores penetraciones, 
con respecto a la media y alta, la parte baja con una baja densidad permite mayor penetración del preservante, en las muestras se identificaron mayores densidades en la media y alta respectivamente.

Los resultados obtenidos en la retención de las sales de boro, son superiores a los establecidos por la (FAO, 1986). Se encuentran entre los niveles aceptables de preservación 1.5 y $2.0 \mathrm{~kg} \mathrm{EAB} / \mathrm{m} 3$ y cumplen con los valores mínimos para que actúe como preservante (1 a $4 \mathrm{~kg} / \mathrm{m3}$, según (Posada Giraldo, 2015). La retención durante 4 o 5 días son diferentes a los sugeridos por (Montoya Arango, 2008) (4 kg/m3 EAB, como retención mínima).

El sistema de uniones en ambas especies con perno con y sin mortero presenta alternativas de uso para esfuerzos mecánicos importantes, el tipo de unión con y sin mortero que resiste más carga máxima es la "P" tensión paralela a la fibra, le sigue la "T" compresión perpendicular a la fibra y finalmente la "Q" tensión perpendicular a la fibra. En la G. aculeata, rellenar los entrenudos con mortero casi duplica la resistencia a diferentes esfuerzos mecánicos. Las propiedades mecánicas del sistema de uniones en ambas especies presentan excelentes condiciones para ser utilizado en sistemas constructivos para la vivienda.

Los resultados de la investigación ofrecen una tecnología con Guadua aculeata, a través de un trabajo sistemático, participativo y de organización, que reduce los costos de la obra, cubre las necesidades espaciales y detona el cambio de una realidad social. El sistema constructivo promueve la prefabricación de elementos estructurales disminuyendo considerablemente el tiempo de ejecución de la obra. Por su facilidad de aprendizaje permite adoptarlo como un sistema de autoconstrucción. La industrialización es posible en elementos portantes como muros de carga, divisorios, puertas, ventanas, muebles, pisos, y recubrimientos de muros. Utilizar el sistema constructivo con bambú Guadua aculeata para la construcción de viviendas siempre tendrá un costo menor que con materiales tradicionales. Es importante utilizar un sistema mixto, que involucre materiales tradicionales. Construir con G. aculeata es una alternativa que produce beneficios ecológicos, económicos y sociales

La propuesta funcional, formal y espacial, es una expresión de aspectos tradicionales, culturales e históricos de la vivienda tradicional totonaca, se rescatan los materiales tradicionales y la sabiduría de los sistemas constructivos ancestrales, con el uso racional de los materiales. Representa el concepto de vivienda "evolucionada" de (Moya Rubio, 1982). El sistema estructural, es un reflejo de la forma natural de trabajo de la G. aculeata, a tensión y compresión, cerchas cuyo origen son las tijeras en la solución de la cubierta de la vivienda tradicional, la geometría responde a cuestiones culturales de funcionamiento y fuerzas físicas. El rescate del bajareque con materiales mixtos que buscan la durabilidad y menor mantenimiento.

La similitud con la vivienda tradicional actual, evoca los valores e identidad que han permanecido a través del tiempo, se conjuga la modernización, tradiciones, disposición de los recursos naturales y la organización espacial.

El crecimiento progresivo de la vivienda a través de la modulación y prefabricación, conduce a la racionalización de los sistemas constructivos, con procedimientos sencillos y fáciles se puede utilizar en la autoconstrucción y disminución de los costos con la participación de la mano de obra solidaria o a mano vuelta.

El prototipo construido es un modelo de solución que puede transformar el contexto circundante con la participación solidaria de la mano de obra y representar una alternativa para la producción social de la vivienda.

\section{AGRADECIMIENTOS}

Los autores agradecen al laboratorio de pruebas de productos maderables de la Red Ambiente y Sustentabilidad del Instituto de Ecología, A.C, de la ciudad de Xalapa, Ver., lugar donde se realizaron los ensayos mecánicos.

Al Instituto de Investigaciones Forestales, Agrícolas y Pecuarias, centro experimental "Las Margaritas" en Hueytamalco, Puebla, por haber proporcionado las muestras para toda la investigación. 


\section{REFERENCIAS}

Colombianas, Normas Técnicas NTC. (2010). Estructuras de Madera y estructuras de Guadua, NSR-10. 156. Colombia.

Cruz Ríos, H. (2013). Bambú Guadua, Guadua aculeata. Estudio, Bamboo Premier, México D.F.

Cruz, L. P. (2018). Determinación del ácido bórico y bórax, usado como preservante en la Guadua aculeata. Tesis licenciatura, Universidad Veracruzana, Facultad de Ciencias Químicas, Poza Rica, Veracruz.

Erdoiza, J., \& Echenique, R. (1980). Preservación de madera de pino con sales de boro. Instituto Nacional de Investigaciones sobre recursos bióticos, Laboratorio de ciencia y tecnología de la madera. Xalapa, Ver.: Instituto Nacional de Investigaciones sobre recursos bióticos.

FAO Department, F. (1986). Wood preservation manual. Rome, Rome, Italy.

GOCDMX, NTC. (2017). Gobierno de la Ciudad de México, Normas técnicas Complementarias Obtenido de Sociedad Mexicana de Ingeniería Estructural, A.C.: http://www.smie.org.mx/informacion-tecnica/normastecnicas-complementarias.php?ntc=ntc-madera

INFONAVIT, C. P. (1999). http://portal.infonavit.org.mx/wps/wcm/connect/inf onavit/el+instituto/biblioteca/ biblioteca_digital_in fonavit. Recuperado el 13 de agosto de 2015,dehttp://portal.infonavit.org.mx/wps/wcm/ connect/infonavit/el+instituto/biblioteca/biblioteca_digital_infonavit:http://infonavit.janium.com/janium-bin/ sumario.pl?ld=20150813123018

Instituto Colombiano de Normas Técnicas y Certificación (ICONTEC). (26 de 03 de 2008). Norma Técnica NTC Colombiana 5300. Bogotá, Bogotá, Colombia.

ISO 22157-1 (2004). International Organization for Standardization. (junio de 2004). Bamboo -- Determination of physical and mechanical properties -- Part 1: Requirements. Geneva, Switzerland.

Londoño , X. (2005). Aspectos generales de los bambúes latinoamericanos. Congreso Mexicano del bambú, I, pág. 3. Xalapa, Ver.

Montoya Arango, J. A., \& Jimenez Arias, E. (6 de enero de 2006). Determinación de la curva de secado al aire libre, mediante modelación matemática y experimental de la Guadua angustifolia Kunth. Scientia et Technica Año XII, No 30, Mayo de 2006 UTP. ISSN 0122-1701, 415-419.

Montoya Arango, J. A., \& Jiménez Arias, E. (6 de enero de 2006). Determinación de la curva de secado al aire libre, mediante modelación matemática y experimental de la Guadua angustifolia Kunth. Scientia et Technica Año XII, No 30, mayo de 2006 UTP. ISSN 0122-1701, 415- 419.

Morán Ubidia, J. A. (S/F). Preservación del bambú $\square$ en América Latina, mediante métodos tradicionales (Vol. 1). (R. I. INBAR, Ed.) Guayaquil, Guayaquil, Ecuador: Red Internacional de Bambú $\square$ y Ratán INBAR.

Norma Técnica NTC Colombiana 5301. (03 de Agosto de 2007). Preservación y secado del culmo de Guadua angustifolia Kunth. Bogotá, Bogotá, Colombia.

Ordoñez Candelaria, V. R., \& Bárcenas Pazos, M. G. (2014). Propiedades Físicas y mecánicas de tres especies de guaduas mexicanas. Maderas y Bosques, 20 (2-III-125), 112-127. 
Ordoñez., M. e. (2012). Investigación para el diseño y construcción con bambú $\square$. Investigación, Instituto de Ecología, A.C., Xalapa, Veracruz.

Peña V., L., Burgo s., A., González F., A., \& Will Valero, S. (julio de 2009). Efecto de la preservación con mezclas de bórax-acido bórico y urea formaldehido sobre las propiedades físico- mecánicas y el ataque de insectos en Guadua angustifolia. Revista Forestal Venezolana, 135- 144

Ponce, D. L. (2010). Universidad Nacional Agraria La Molina. Facultad de Ciencias Forestales Características de Preservación por el Método de Inmersión del Tallo de Guadua angustifolia Kunth (bambú), proveniente del Distrito de La Florida, Cajamarca. Tesis Licenciatura, Universidad Nacional Agraria La Molina, Facultad de Ciencias Forestales, Lima, Perú.

Posada, Giraldo, R. E. (2015). Deasrrollo de métodos alternativos de valoración de la calidad de la preservación, empleando sales de boro en la Guadua angustifolia Kunt. Tesis de Maestría, Universidad Tecnológica de Pereira, Facultad de Ciencias Ambientales, Pereira, Colombia.

Turner, A. A. (2008). PENETRATION DEPTH OF BORATES IN HISTORIC WOODEN STRUCTURES IN VIRGINIA CITY, MONTANA. tesis, Faculties of the University of Pennsylvania, $\mathrm{h} \mathrm{p}: / /$ repository.upenn.edu/hp_theses/115, Pennsylvania.

Zaragoza, Hernández, I. (2012). Anatomía y propiedades físico-mecánicas del culmo de bambú $\square$ (Guadua aculeata Rupr.) de la región nororiental del Estado de Puebla. Científico, Universidad Autónoma de Chapingo, División de Ciencias Forestales, Texcoco, Edo. de México.

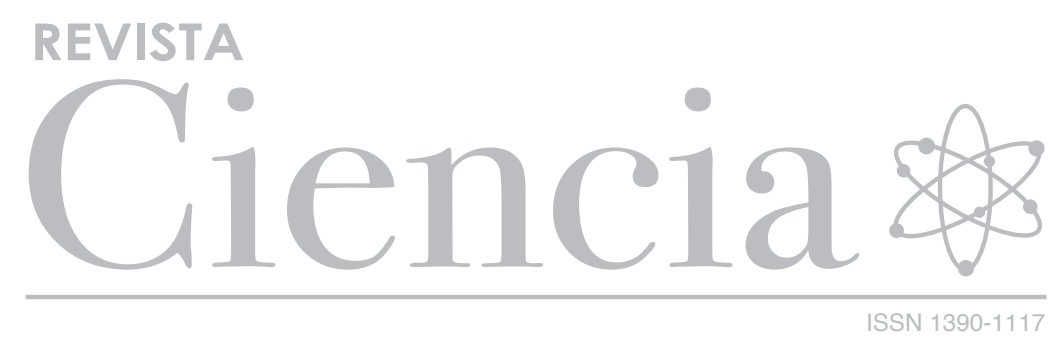

(C) Universidad de las Fuerzas Armadas ESPE Sangolquí, Ecuador.

CIENCIA - QUITO, Volumen 22, No. 1, Enero 2020 